\title{
Application of mass spectrometry to characterize the components present in a curcumin Sample
}

\author{
R.V. Coorey ${ }^{\mathrm{a}}{ }$, and P. Håkansson ${ }^{\mathrm{b}}$ \\ ${ }^{a}$ Department of Physics, University of Colombo, Colombo-03, Sri Lanka \\ ${ }^{b}$ Division of Radiation Sciences, Department of Ion Physics, Uppsala University, Sweden
}

\begin{abstract}
The identification of the components present in crude samples purely by mass spectrometric techniques has been a long-standing problem. Conventionally, components present in a crude sample are isolated and purified by chromatographic methods and then various spectrometric techniques (including mass spectrometric techniques) are adopted for their structure elucidation and physical characterization. This paper gives an example illustrating the characterization of the components present in a crude sample purely by mass spectrometric techniques.
\end{abstract}

A commercially obtained curcumin sample (Assay: 65-70\%) was chosen for this purpose. The mass analysis of this sample using time-of-flight reflectron mass spectrometric techniques namely plasma desorption (PD), laser desorption (LD) and electrospray ionization (ESI) gave evidence for the presence of curcuminoids other than curcumin in the sample. The electrospray Fourier transform ionization cyclotron resonance mass spectrometry combined with tandem mass spectrometry (MS/MS) has been adopted successfully to confirm the presence of all curcuminoids in the curcumin sample.

\section{INTRODUCTION}

A fundamental aim in any drug development process is the identification of all minor components present in a chemically isolated active component of a natural product substance. The minor components that are present at a level of $0.1 \%$ or greater relative to the active component require characterization to comply with regulatory requirements ${ }^{1,2}$. The identification of the components present in a crude sample purely by mass spectrometric techniques has been a long-standing problem. Conventionally, the components present in a crude sample are first isolated and purified by chromatographic methods and then various spectroscopic techniques are adopted for their structure elucidation and physical characterization. However due to the low sensitivity of some spectroscopic methods, (e.g. X-ray crystallography and NMR), this procedure requires the

\footnotetext{
*Correspondence Author, Email: ramal@phys.cmb.ac.lk
} 
isolation of relatively large sample quantities and the expenditure of a significant amount of time and resources.

In contrast, mass spectrometry is a highly sensitive technique requiring only small amounts of material (typically $1 \mu \mathrm{g}$ ) for analysis. The process of elucidating the mass and the structure of a substance can be expedited by using a high performance mass spectrometer. With the advent of "soft" ionization mass spectrometric techniques such as plasma desorption (PD) ${ }^{4}$, laser desorption $(\mathrm{LD})^{5}$, and electrospray ionization $(\mathrm{ESI})^{6}$ that produce protonated molecular ions with minimal or no fragmentation, pseudo-molecular ions of each eluting species can be readily produced. The ESI mass spectrometry ${ }^{6}$ is superior to others in this respect since structurally significant information can be elicited by collisional activation of the eluting species in the vacuum interface of the ESI source. However, to ensure that the information obtained is indeed related to the compound in question, it will be important to isolate the precursor ion for the analysis by MS/MS ${ }^{7}$.

Fourier transform ion cyclotron resonance mass spectrometry (FTICR-MS) ${ }^{8,9}$ can readily provide high resolution, exact mass analysis. Furthermore, FTICR-MS could be used to perform multiple stages of mass analysis, (i.e $\mathrm{MS}^{\mathrm{n}}$ ) by isolating the precursor ion and generating fragment ions by using collisional induced dissociation ${ }^{10}$ in successive fashion. Therefore, unlike other forms of mass spectrometry, ESI-FTICR-MS can routinely provide exact mass measurements for all ions, (i.e., precursor and fragments) at each stage of mass analysis.

This paper demonstrates applicability of mass spectrometric techniques to characterize the components present in a commercially obtained crude sample without any further purification of the sample.

\section{EXPERIMENTAL}

\section{Test sample}

1,7-bis[4-hydroxy-3-methoxy-phenyl]-1,6-heptadiene-3,5-dione or popularly known as curcumin-I (or curcumin) (368 u) is the main ingredient present in turmeric, (Curcuma longa L) which is mainly used as a spice in food. Turmeric possesses anti-fungal, antibacterial properties and it is used for arthritis, high cholesterol, digestion, liver protection, and obesity ${ }^{11}$. Therefore it is a substance of pharmaceutical interest. Curcumin is one of the three curcuminoids present in turmeric. Gas chromatographic and mass spectrometric studies have confirmed the structures and the molecular weights of the other two curcuminoids, which are curcumin-II or desmethoxycurcumin [4-hydroxy-cinnamoyl(4-hydroxy-3-methoxy-cinnamoyl) methane] $(338$ u) and curcumin-III or bisdemethoxycurcumin [bis-(4-hydroxy-3-methoxy-cinnamoyl) methane] (308 u) (Fig. $1)^{12}$. 


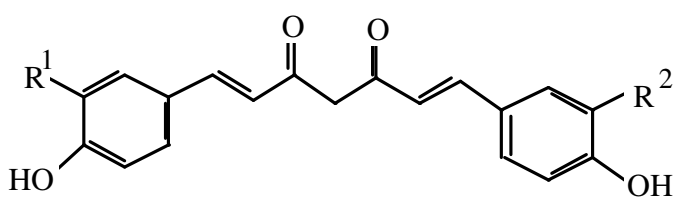

Figure 1. The chemical structure of curcuminoids. Curcumin-I (Curcumin) has $R^{1}=R^{2}=O M e$, curcumin-II (desmethoxycurcumin) has $\mathrm{R}^{1}=\mathrm{OMe} \& \mathrm{R}^{2}=\mathrm{H}$ and curcumin-III (bisdemethoxycurcumin) $\operatorname{hasR}^{1}=\mathrm{R}^{2}=\mathrm{H}$

For the present study, a curcumin sample (Assay: 65-70\%) was purchased from Sigma (Stockholm, Sweden) (Product No: C1386) and used without further purification.

\section{Sample preparation}

For the PD mass analysis, the curcumin sample was dissolved in acetone (concentration $\sim 1 \mu \mathrm{g} / \mu \mathrm{L}$ ) and electrosprayed $200 \mu \mathrm{L}$ of each solution on to an aluminized Mylar backing. For the LDI mass analysis, the curcumin sample was dissolved in acetone (concentration $\sim 1 \mu \mathrm{g} / \mu \mathrm{L}$ ). A solution of $1 \mu \mathrm{l}$ was dried on a stainless steel sample backing. For the ESI mass analysis, the curcumin sample was dissolved in a 1:1 methanol/acetic acid and $0.1 \% \mathrm{HCl}$ buffer (conc. $10^{-6} \mathrm{M}$ ) solution. For ESI a sample of Substance P, purchased from Sigma-Aldrich (Stockholm, Sweden), was prepared in the similar procedure to that of curcumin.

\section{Mass spectrometry}

\section{(a) Plasma desorption mass spectrometry}

Positive ion PD mass spectra of the curcumin sample were accumulated using a time-offlight (TOF) instrument BioIon 20 (BioIon AB, Sweden) incorporating an Einzel lens and an electro-static reflector. The data acquisition was done using a TDC and was recorded on an ATARI computer with the software program TOFMA. Mass calibration was performed using the monoisotopic masses of $\mathrm{H}^{+}$and $\mathrm{CH}_{3}{ }^{+}$ions.

\section{(b) Laser induced mass spectrometry}

The LDI mass spectrum of the curcumin sample was collected using a TOF, singlestage ion reflector instrument, Uppsala, Sweden using standard 337-nm nitrogen laser with delayed ion extraction ${ }^{13}$ conditions. The data acquisition was done using a digital oscilloscope and the mass analysis was done with the software program 'ULISSES 7.3'. Fifteen laser shots were averaged in order to improve the signal-to-noise ratio. External calibration of the mass spectrum was performed. 


\section{(c) Electrospray ionization mass spectrometry}

The ESI-TOF mass spectra of the curcumin sample were obtained using a TOF, single-stage electrostatic reflector instrument, Uppsala, Sweden. ${ }^{14}$ Positive ion mass spectra were achieved by infusing $10^{-6} \mathrm{M}$ solutions of compounds (sample and the buffer) into the electrospray ion source at $0.1 \mu \mathrm{l} / \mathrm{min}$ by means of a syringe pump. The spray needle voltage was set at $+5 \mathrm{kV}$. The whole atmosphere vacuum interface was floated on the acceleration voltage of $+3 \mathrm{kV}$. The orthogonal extraction was performed by two extraction pulses of reverse polarity (i.e. $+145 \mathrm{~V}$ for the positive pulse and $-136 \mathrm{~V}$ for the negative pulse). The reflecting voltage was set at $+3.5 \mathrm{kV}$. The data acquisition was done using a TDC and was recorded on an ATARI computer with the software program TOFMA External calibration of ESI mass spectra was performed using the monoisotopic peaks of substance $\mathrm{P}$ using the conventional TOF equation (2-point calibration).

The ESI positive ion mass spectra of the curcumin sample were also accumulated using the commercially available FT-ICR instrument, Bruker (Billerica, MA, USA) Bio APEX-94e with a 9.4 Tesla superconducting magnet. A FT-ICR-MS/MS study of the curcumin sample was performed using the SORI-CID method ${ }^{11}$ in order to perform sequential activation of ions by low energy collisions with nitrogen gas.

\section{RESULTS AND DISCUSSION}

\section{Time-of-flight mass analysis of the curcumin sample}

The TOF mass analysis of the curcumin sample by PD, LD, and ESI techniques has yielded the $\mathrm{MH}^{+}$ion of curcumin (Fig. 2). The major fragment ion of curcumin, $\mathrm{m} / \mathrm{z} 177$ is observed with all these techniques (Fig. 3). Many other fragment ions peaks of curcumin, $\mathrm{m} / \mathrm{z}$ 137, 149, 191, 219 and 298 are also observed in the PD-TOF mass spectrum [Fig. 3 (a), Table 1]. Some of these fragment ion peaks are also observed in the LD- and ESI-TOF mass spectra [Fig. 3 (b,c), Table 1]. All these mass assignments agree with the mass data of electron impact ionization mass spectrum of curcumin. ${ }^{15}$ 

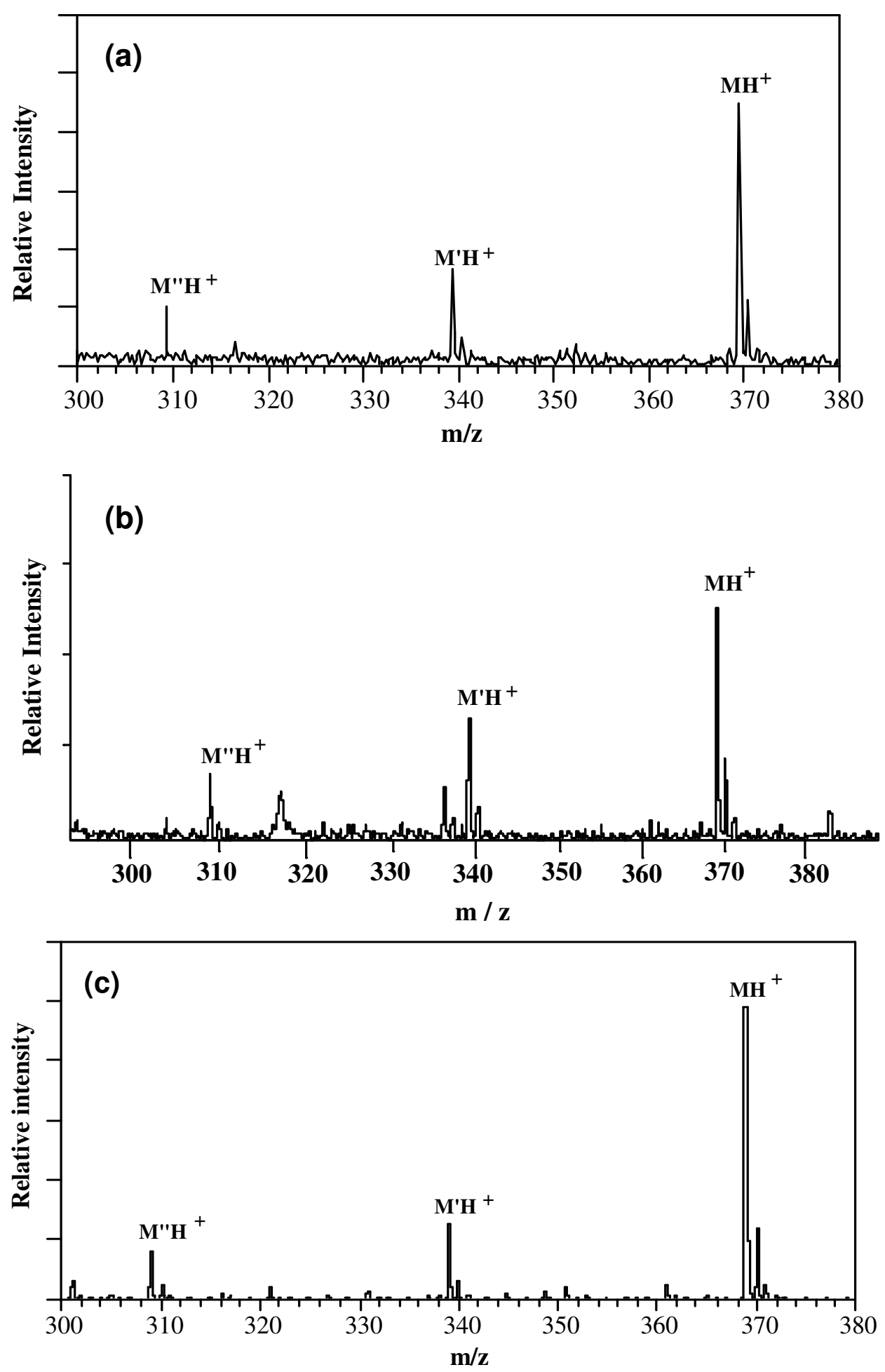

Figure 2. (a) PD- (b) LDI- and (c) ESI- TOF mass spectra of the curcumin sample in the mass range 300 $380 \mathrm{Da}$. Each technique gives the $\mathrm{MH}^{+}$peak of curcuminoids. 

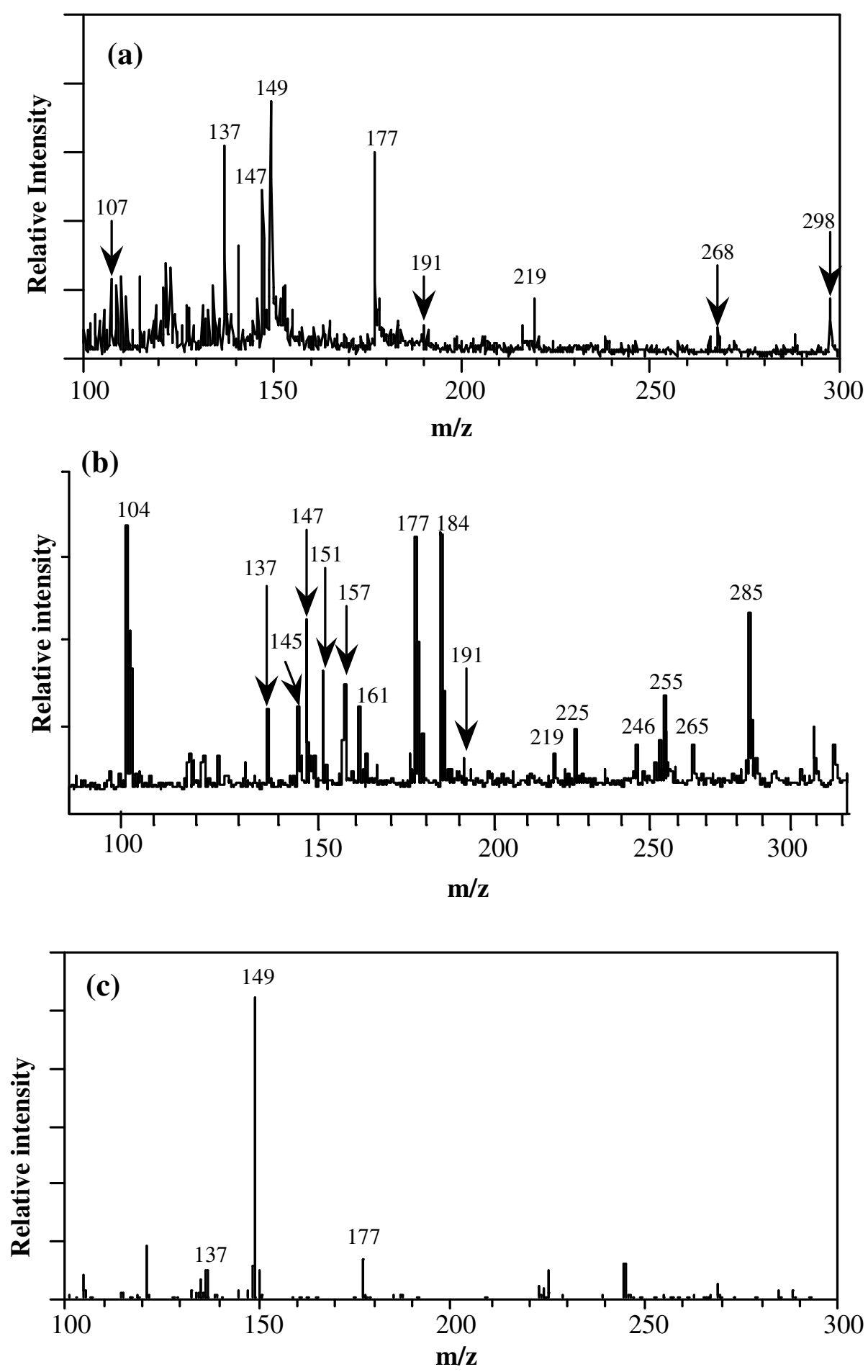

Figure 3. (a) PD- (b) LD- and (c) ESI-TOF mass spectra of the crude curcumin sample in the mass range 100 - $300 \mathrm{Da}$. In addition to the fragment ion peaks of curcumin, some fragment ion peaks of other curcuminoids are also observed in the PD- and LD- and ESI-TOF mass spectra. 
Table 1. The peaks (m/z) in the PD mass spectrum of the curcumin sample are compared with the EI mass assignments of individual curcuminoids

\begin{tabular}{|c|c|c|c|c|}
\hline \multirow{2}{*}{$\begin{array}{l}\text { Peaks }(\mathrm{m} / \mathrm{z}) \text { in the PD mass } \\
\text { spectrum of the curcumin sample }\end{array}$} & \multicolumn{3}{|c|}{ Mass assignments in the EI mass spectrum (as given in ref. 15) } & \multirow{2}{*}{$\begin{array}{c}\text { Elementary } \\
\text { composition of ions }\end{array}$} \\
\hline & curcumin & desmethoxycurcumin & bisdemethoxycurcumin & \\
\hline 369 & 369 & - & - & $\mathrm{MH}^{+}$ \\
\hline 339 & - & 339 & - & $\mathrm{M}^{\prime} \mathrm{H}^{+}$ \\
\hline 309 & - & - & 309 & $\mathrm{M}^{\prime} \mathrm{H}^{+}$ \\
\hline 298 & 298 & & & {$\left[\mathrm{M}-\mathrm{C}_{3} \mathrm{H}_{2} \mathrm{O}_{2}\right]^{+}$} \\
\hline 268 & & 268 & & {$\left[\mathrm{M}^{\prime}-\mathrm{C}_{3} \mathrm{H}_{2} \mathrm{O}_{2}\right]^{+}$} \\
\hline 219 & 219 & & & $\begin{array}{l}{\left[\mathrm{M}-\mathrm{C}_{9} \mathrm{H}_{9} \mathrm{O}_{2}\right]^{+}} \\
{\left[\mathrm{M}^{\prime}-\mathrm{C}_{8} \mathrm{H}_{7} \mathrm{O}\right]^{+}}\end{array}$ \\
\hline 191 & 191 & & & $\begin{array}{l}{\left[\mathrm{M}-\mathrm{C}_{10} \mathrm{H}_{9} \mathrm{O}_{3}\right]^{+}} \\
{\left[\mathrm{M}^{\prime}-\mathrm{C}_{9} \mathrm{H}_{7} \mathrm{O}_{2}\right]^{+}}\end{array}$ \\
\hline 177 & 177 & 177 & & $\begin{array}{c}{\left[\mathrm{M}-\mathrm{C}_{11} \mathrm{H}_{11} \mathrm{O}_{3}\right]^{+}} \\
{\left[\mathrm{M}^{\prime}-\mathrm{C}_{10} \mathrm{H}_{9} \mathrm{O}_{2}\right]^{+}}\end{array}$ \\
\hline 149 & 149 & & & $\begin{array}{c}{\left[\mathrm{M}-\mathrm{C}_{12} \mathrm{H}_{11} \mathrm{O}_{4}\right]^{+}} \\
{\left[\mathrm{M}^{\prime}-\mathrm{C}_{11} \mathrm{H}_{9} \mathrm{O}_{3}\right]^{+}}\end{array}$ \\
\hline 147 & & 147 & 147 & $\begin{array}{l}{\left[\mathrm{M}^{\prime}-\mathrm{C}_{11} \mathrm{H}_{11} \mathrm{O}_{3}\right]^{+}} \\
{\left[\mathrm{M}^{\prime \prime}-\mathrm{C}_{10} \mathrm{H}_{9} \mathrm{O}_{2}\right]^{+}}\end{array}$ \\
\hline 137 & 137 & & & $\begin{array}{c}{\left[\mathrm{M}-\mathrm{C}_{13} \mathrm{H}_{11} \mathrm{O}_{4}\right]^{+}} \\
{\left[\mathrm{M}^{\prime}-\mathrm{C}_{12} \mathrm{H}_{9} \mathrm{O}_{3}\right]^{+}}\end{array}$ \\
\hline 107 & - & 107 & 107 & $\begin{array}{l}{\left[\mathrm{M}^{\prime}-\mathrm{C}_{13} \mathrm{H}_{11} \mathrm{O}_{4}\right]^{+}} \\
{\left[\mathrm{M}^{\prime \prime}-\mathrm{C}_{12} \mathrm{H}_{9} \mathrm{O}_{3}\right]^{+}}\end{array}$ \\
\hline
\end{tabular}


The PD-, LD-, and ESI-TOF mass spectra of the curcumin sample (Fig. 2) display the probable $\mathrm{MH}^{+}$peaks of desmethoxycurcumin $\left(\mathrm{M}^{\prime} \mathrm{H}^{+}: \mathrm{m} / \mathrm{z}\right.$ 339) and bisdemethoxycurcumin $\left(\mathrm{M}^{\prime \prime} \mathrm{H}^{+}: \mathrm{m} / \mathrm{z}\right.$ 309). According to the electron impact ionization mass spectrum of curcumin ${ }^{15}$ these peaks could not have arisen due to the fragments of curcumin. Therefore it implied that the presence of other curcuminoids in the curcumin sample might have given rise to these peaks. The presence of peaks at m/z 107, 147 and 268 that observed in the curcumin mass spectrum co-related to the fragment ion peaks of these curcuminoids (Fig. 2). For example the peaks at m/z 107 and 147 correspond to the probable fragment ions respectively of either desmethoxycurcumin or bisdemethoxycurcumin (Table 1). The peak at $\mathrm{m} / \mathrm{z} 268$ corresponds to the probable fragment of desmethoxycurcumin. These results gave further evidence for the presence of curcuminoids in the curcumin sample.

The LD mass analysis of the curcumin sample there exists some additional peaks, which are not present in the PD or ESI mass spectra of the curcumin sample. None of the recorded electron impact ionization mass spectra of curcumin or other curcuminoids ${ }^{15}$ show the additional peaks observed in the LD mass spectrum of the curcumin sample. Thus it appears that these peaks may originate due to photochemical degradation undergone by curcumin under UV conditions. ${ }^{2}$ However, in the present instance no effort is taken to identify these peaks.

\section{The Fourier transform ion cyclotron resonance mass analysis of the curcumin sample}

The ESI-FTICR mass spectrum of the curcumin sample gave the $\mathrm{MH}^{+}$peak of curcumin at $\mathrm{m} / \mathrm{z} 369$ and the peaks at $\mathrm{m} / \mathrm{z} 339\left(\mathrm{M}^{\prime \prime} \mathrm{H}^{+}\right)$, and $309\left(\mathrm{M}^{\prime} \mathrm{H}^{+}\right)$due to the probable curcuminoids present in the curcumin sample [Fig. 4(a)]. The FT-ICR MS/MS spectra of curcumin (isolated from other curcuminoids) and each of the other probable curcuminoids namely desmethoxycurcumin and bisdemethoxycurcumin (isolated from other curcuminoids) are shown in Fig.3 (b,c,d). The MS/MS ESI-FT-ICR mass spectrum of curcumin (isolated from other curcuminoids) shows the $\mathrm{MH}^{+}$peak $(\mathrm{m} / \mathrm{z} 369)$ and the fragment at m/z 177 but a peak at m/z 147 is not observed in this spectrum. This indicates that the fragment at $\mathrm{m} / \mathrm{z} 147$ is not due to curcumin [Fig. 4(b)]. The ESI-FT-ICR MS/MS spectrum of desmethoxycurcumin (isolated from other curcuminoids) shows the $\mathrm{M}^{\prime} \mathrm{H}^{+}$peak (at m/z 339) with fragment ions at m/z 147 and 177 [Fig. 4(c)]. The ESI-FT-ICR-MS/MS spectrum of bisdemethoxycurcumin (isolated from other curcuminoids) shows the $\mathrm{M}^{\prime \prime} \mathrm{H}^{+}$ peak (at $\mathrm{m} / \mathrm{z}$ 309) and the fragment ion at $\mathrm{m} / \mathrm{z} 147$ but a peak at $\mathrm{m} / \mathrm{z} 177$ is not observed in this spectrum [Fig. 4(d)]. These results show a definite confirmation of the presence of curcuminoids in the curcumin sample. The observed peaks in the ESI-FT-ICR MS/MS spectra of curcumin agree very well with the structures of these curcuminoids (Fig. 1). The other peaks present in the FT-ICR mass spectra (e.g. peak at m/z 145) may be due to the system (probably due to noise signals). 

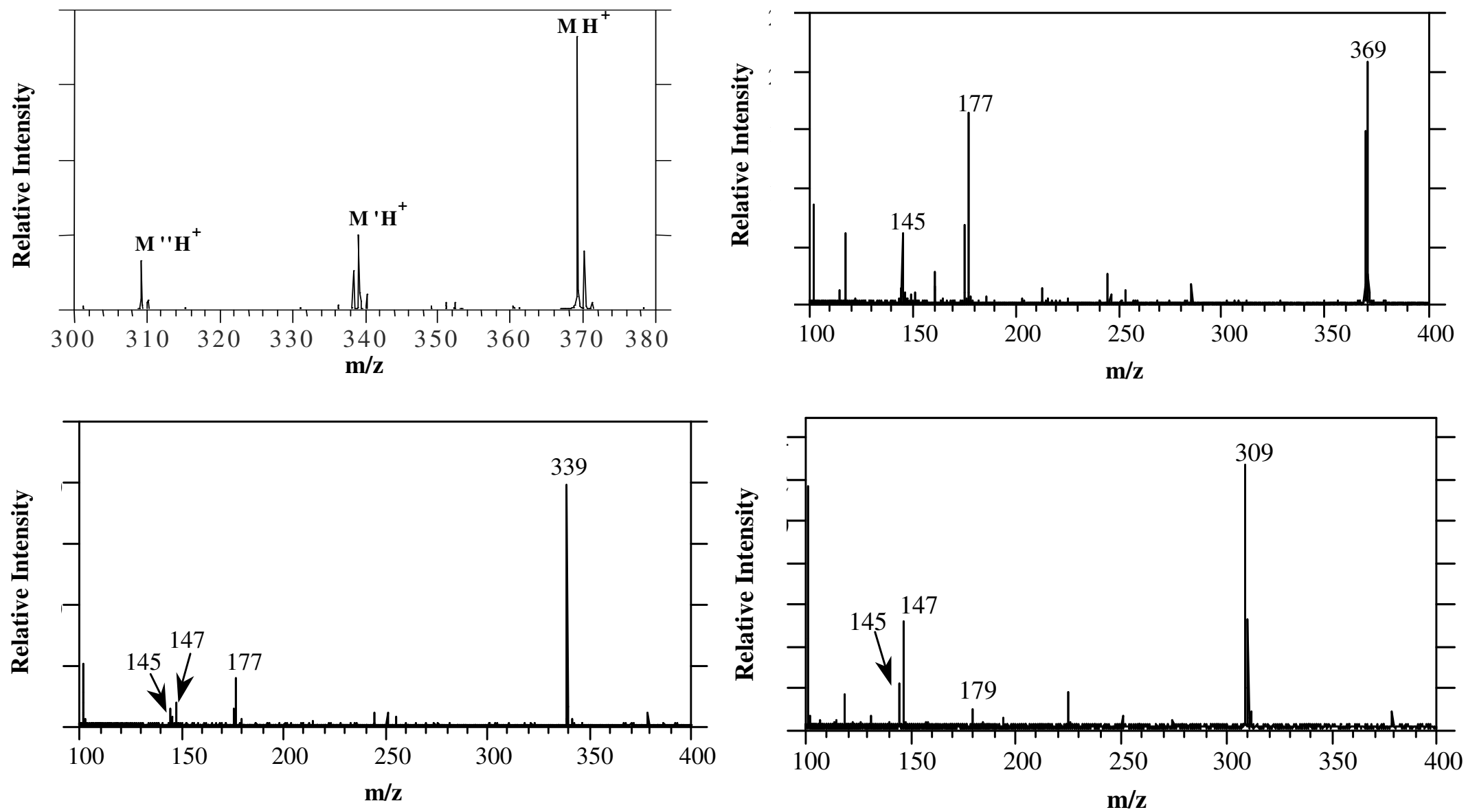

Figure 4. (a) ESI-FT-ICR positive ion mass spectrum of the curcumin sample is shown. $\mathrm{MH}^{+}$peak of curcumin and other curcuminoids are observed. FT-ICR- MS/MS spectra of the isolated $\mathrm{MH}^{+}$ion and fragments of (b) curcumin (369), (c) demethoxy curcumin (339) and (d) bisdemethoxy curcumin (309) are also shown. 


\section{CONCLUSIONS}

A curcumin sample commercially obtained from Sigma (Stockholm, Sweden) was mass analyzed using time-of-flight reflectron mass spectrometry with PD, LDI and ESI techniques. All these techniques gave evidence for the presence of curcuminoids other than curcumin in the sample. An MS/MS study using the ESI-FT-ICR-MS has been used successfully to confirm the presence of these curcuminoids in the curcumin sample.

\section{ACKNOWLEDGEMENTS}

Financial support provided for RVC from International Science Programs, Uppsala University, Sweden under Grant No. SRI 01/2 is gratefully acknowledged. RVC also thanks Kristina Håkansson and Magnus Palmbald for the help given to operate the ESITOF and FT-ICR instruments.

\section{REFERENCES}

1. International Conference on Harmonisation; Guideline on Impurities in New Drug Substances, Fed. Register 1996; 61 (3): 371

2. International Conference on Harmonisation; Guideline on Impurities in New Drug Products, Fed. Register 1997; 62 (96): 27454

3. Mamyrin BA, Karatajev VJ, Shmikk, DV and Zagulin VA. Soviet Phys. JETP 1973; 37: 45

4. Torgerson DF, Skowronski RP and MacFarlane RD Biochem. Biophys. Res. Commun. 1974; 60: 616

5. Posthumus MA, Kistmaker PG, Meuzelaar HLC and Ten Nover de Brauw MC. Anal.Chem 1978; 50: 985

6. $\quad$ Yamashita M and Fenn JB. J. Phys. Chem. 1984; 88: 4451

7. McLafferty, F.W. (Ed.) Tandem Mass Spectrometry, Wiley, New York, 1983

8. Comisarow MB and Marshall AG. Chem.Phys. Lett 1974; 25: 282

9. Comisarow MB. Adv. Mass Spectrom 1980; 8: 1698

10. Gauthier JW, Trautman RR and Jacobson, DB. Anal. Chem. Acta 1991; 246: 211

11. Fisher C. Phenolic Compounds in Spices, ACS Symposium Series.1992; 506: 118

12. Tфnnesen HH and Karlsen J. Institute of Pharmacy, University of Oslo, Norway 1986

13. Vestal ML, Juhasz P and Martin SA. Rapid Commun. Mass Spectrom. 1995; 9: 1044

14. Håkansson K, Zubarev RA, Håkansson P, Laiko V and Dodonov AF. Rev. Sci. Instr.2000; 71: 36

15. Berzin, VB, Katsitadze, LG, Pilipenko, TV, Ovcharenko, VV and Miroshnikov, AI. Russian Journal of Bioorganic Chemistry 1996; 22: 696 